\title{
Improvement of well-being in cancer patients by yoga training
}

\author{
Jörg Haier, ${ }^{1}$ Antje Duda, ${ }^{2}$ Claudia Branss-Tallen ${ }^{2}$ \\ ${ }^{1}$ Healthcare Management, Nordakademie Graduate School, Hamburg, Germany \\ ${ }^{2}$ Comprehensive Cancer Center Münster, University Hospital Münster, Germany
}

\section{ABSTRACT}

Background: Cancer patients frequently suffer from a reduced physical activity. Non-pharmacological interventions, such as yoga, provide opportunities to deal with these symptoms. The aim of this study is to evaluate the effect of yoga on the well-being of cancer patients.

Methods: Between 2015 and 2017, we analyzed the effects of yoga on the well-being of cancer patients. Fifty-one patients received yoga training specifically designed to address cancer-related problems. Before the start (SoS) and after the end (EoS) of training, the WHO-5 well-being index was used to evaluate patients' perception.

Results: WHO-5 scores significantly improved from $47.1 \pm 21.4$ at SoS to $69.7 \pm 15.1$ at EoS. Patients in the middle age group (EoSSoS: $28.0 \pm 20.6)$ showed a significantly higher benefit $(\mathrm{p}<0.005)$ compared with age groups < 40 years $($ EoS - SoS: $10.7 \pm 15.4)$ and $>60$ years (EoS-SoS: 16.0 12.1$)$. Patients with low initial scores benefited most from yoga.

Conclusion: Our study showed that yoga training under experienced supervision could serve as a beneficial complementary treatment in cancer patients. Best effects were observed in patients with highly reduced well-being and 40-60-year-old patients.

Keywords: cancer, fatigue, non-pharmacological interventions, physical activity, WHO-5 well-being index, yoga pISSN: 0853-1773• eISSN: 2252-8083・https://doi.org/10.13181/mji.v27i3.2744 • Med J Indones. 2018;27:185-93

- Received 27 Mar 2018• Accepted 6 Sep 2018

Corresponding author: Jörg Haier

joerg.haier@nordakademie.de

Copyright @ 2018 Authors. This is an open access article distributed under the terms of the Creative Commons Attribution-NonCommercial 4.0 International License (http://creativecommons.org/licenses/by-nc/4.0/), which permits unrestricted non-commercial use, distribution, and reproduction in any medium, provided the original author and source are properly cited. 
Cancer-related fatigue and dysthymia interfere with various physiological functions causing and increasing a physical, emotional, and cognitive exhaustion. Non-pharmacological interventions to deal with these symptoms include psychosocial interventions, physical exercise, yoga, physiotherapy, dietary management, among others. ${ }^{1}$ Within this diverse group, physical activities have been identified as a potential intervention in cancer patients, which has been mainly investigated in women with breast cancer. This approach seems to be an effective way to improve quality of life, increase physical activity, and reduce fatigue in these patients. ${ }^{2}$ One opportunity to perform such exercises appears to be yoga, but most investigations come from its cultural origins, such as India ${ }^{3}$ and Japan, ${ }^{4}$ and this cross-cultural perception might influence its adoption. ${ }^{5}$ Yoga is performed as mind-body exercises, a combination of physical poses with breathing and meditation. ${ }^{6}$ This type of training has been associated with a reduction in evening cortisol, waking cortisol, ambulatory systolic blood pressure, resting heart rate, high-frequency heart rate variability, fasting blood glucose, cholesterol, and low-density lipoprotein..$^{7-9}$

Recently, it have shown that practicing yoga during chemotherapy may result in modest short-term benefits in sleep quality and additional long-term benefits may emerge over time in breast cancer patients. ${ }^{10}$ Similarly, yoga appears to reduce anxiety in these women. ${ }^{11}$ However, yoga-related benefits have been almost exclusively investigated in women with breast cancer. ${ }^{12,13}$ Effects on patients with other types of cancer have been less examined. For example, during radiotherapy, yoga was also associated with a significant reduction in fatigue, and both urinary and sexual dysfunction in prostate cancer patients. ${ }^{14}$ In glioma patients, quality of life was moderately improved. ${ }^{15}$

Although positive effects have been suggested, many cancer patients perceive barriers to certain types of exercise. ${ }^{2}$ This issue relates not only to physical discomfort and feeling sick, but also to low mood, selfconsciousness, fatigue, and fear of overdoing it. $^{16-18}$ Several essential factors contribute to and result in this patient behavior including the lack of sufficient information, the absence of specific and trusted supervision for training cancer patients, and misleading expectations of patients and/or trainers.

Overall, the inclusion of mind-bodyapproaches in cancer care is promising. Also, evidence of efficacy for treating many common symptoms is increasing mainly for breast cancer patients. ${ }^{19-21}$ Therefore, Carlson et $\mathrm{al}^{22}$ requested in their recent review that future studies should investigate more diverse cancer populations using standardized treatment protocols. Our investigation targeted this issue and analyzed the effects of yoga on the well-being of patients with a wide range of cancer types and during various stages of treatment.

\section{METHODS}

\section{Patients}

Between January 2014 and December 2016, all cancer patients at the Comprehensive Cancer Center were informed about the opportunity to participate in the yoga trial by written information material. Interested patients had to contact the trial group to become enrolled. Patients who provided informed consent (Joint Ethics Committee of Physicians Chamber Westfalen-Lippe and Wilhelms-University Muenster, Germany: 2014-636-f-S) were included in two subsequent training groups. Each group performed 10 yoga sessions at the beginner level without the requirement of preexisting knowledge or experience in yoga. Patients had to be older than 18 years without the limitations of specific cancer diagnoses. Initial assessment only concentrated on the general physical ability to participate in yoga exercises.

\section{Treatment}

Each course lasted 90 minutes and was limited to 10 participants. The training concept was specifically composed to consider both the physical and emotional limitations of cancer patients. Lessons started with relaxation, breathing exercises, and attention to one's own body. Subsequently, light power training combined with body reception was done in which the intensity was adapted to the cancer history of each patient. For example, in breast cancer patients stretching of the thorax was the focus whereas patients with prostate or gynecological cancer were oriented toward perineal exercises. 
Frequently, resting and cool-down phases were included. Lessons were finished with a foot massage (especially in cases of polyneuropathy) and meditation. More intensive yoga exercises, such as handstands, headstands, inverse or extreme body positions, and intensive breathing techniques, were omitted entirely.

\section{Evaluation of effects}

Various questionnaires have been considered for potential evaluation of training effects on quality of life and well-being of cancer patients. The daily fatigue impact scale (D-FIS) is focused toward fatigue, which was considered as too narrow in one direction for the study's purposes. The treatment satisfaction with medicines (STATMED-Q) asks for side effects, but not primary treatment results. The symptom impact questionnaire (SIQR) measures physical activities, but not emotional factors. The sickness impact profile and short form health survey (SF36) are quite long and not suitable for repetitive usage in a training environment for patients. Therefore, the short and easy to use 5-item World Health Organization Well-Being Index (WHO-5) has been used for quantification of well-being. This instrument is among the most widely used questionnaires to assess subjective psychological well-being. This wellbeing index includes both negative and positive aspects of well-being in a single unidimensional scale during the last two weeks before rating the aspects. Its advantage is the ability to show the overall change along the continuum of wellbeing, thus facilitating comparisons between both patient groups and treatments. The WHO5 has high clinimetric validity, can be used as an outcome measure balancing the wanted and unwanted effects of treatments, is a sensitive and specific screening tool for depression, and has very high applicability across fields of study. ${ }^{23}$ It is not specific to any disease and has been applied as a disease-independent index of well-being in a broad range of healthcare studies.

For this study, we used the German translation of the original questionnaire. The evaluation has been done before the first yoga lesson (start of sessions: SoS) started and at the end of the 10-session (EoS) training period. Patients were asked to indicate over the past 2 weeks: (1) I have felt cheerful and in good spirits, (2) I have felt calm and relaxed, (3) I have felt active and vigorous, (4) I woke up feeling fresh and rested, and (5) my daily life has been filled with things that interest me. Responses to questions had the following rating options "All of the time" (5 points), "Most of the time" (4 points), "More than half the time" ( 3 points), "Less than half the time" ( 2 points), "Some of the time" (1 point), or "At no time" (0 points). Answers were scored according to the instrument's instructions, and final scores were obtained that ranged from 0 representing the worst imaginable well-being to 100 representing the best imaginable well-being. ${ }^{24}$

\section{Statistics}

Variables are expressed as the median/ range or mean \pm standard deviation $(\mathrm{x} \pm \mathrm{SD})$. Student's t-test and Fisher's exact test were used for evaluation of parameters when appropriate. Two-sided approaches and dependent testing were applied for longitudinal comparisons, whereas groups were compared using independent sampling. A Kendall's Tau test was also performed to compare parameters between different patient groups. P-values $<0.05$ were considered significant. All statistical analyses were performed using SPSS 23.0 software package (SPSS, Chicago, Illinois, USA).

\section{RESULTS}

\section{Patient cohort}

Overall, 51 patients ( 44 females, 7 males) were enrolled in the training sessions. Thirty-three percent of patients performed the sessions during continuous cancer-specific treatment whereas the remaining patients performed the sessions after treatment $(48 \%)$ or in rehabilitation immediately after hospital discharge (16\%). Fourty-eight patients provided complete questionnaires at SoS, and 31 patients completed the entire 10 -session training program and then filled in both questionnaires in an evaluable manner. During the training period, 6 patients did not complete yoga training because of new cancer progression. The remaining patients with incomplete courses continued their rehabilitation in a home environment, but none of them declared yoga participation as the reason for dropping out of the follow up. Patients had different cancer diagnoses, and more than $50 \%$ had breast cancer (Table 1). Table 2 showed the demographic data of this study. 
Table 1. Diagnoses of enrolled patients

\begin{tabular}{lc}
\hline Diagnosis & Number of enrolled patients, $\mathrm{n}(\%)$ \\
\hline Bladder & $2(3.9)$ \\
Breast & $30(58.8)$ \\
CNS cancer & $3(5.9)$ \\
Head \& neck & $1(2.0)$ \\
Hematological & $4(7.8)$ \\
Prostate & $2(3.9)$ \\
Sarcoma & $6(11.8)$ \\
Skin cancer & $1(2.0)$ \\
Thyroid & $1(2.0)$ \\
Unknown & $1(2.0)$ \\
\hline Total & $51(100)$ \\
\hline
\end{tabular}

Table 2. Demographic data

\begin{tabular}{|lc}
\hline Variable & Patients \\
\hline Gender, $\mathrm{n}(\%)$ & $44(86.2)$ \\
\hline Female & $7(13.8)$ \\
\hline$\quad$ Male & \\
\hline Age, (years) & $51.8 \pm 9.2$ \\
\hline Mean \pm SD & $32-69$ \\
\hline Range & \\
\hline Treatment status, $\mathrm{n}(\%)$ & $17(33.0)$ \\
\hline $\begin{array}{l}\text { During active treatment } \\
\text { After treatment }\end{array}$ & $24(47.0)$ \\
\hline Early rehabilitation & $8(15.7)$ \\
\hline Yoga training, $\mathrm{n}(\%)$ & $33(64.6)$ \\
\hline $\begin{array}{l}\text { Complete entire } 10 \text { sessions } \\
\text { Incomplete due to progression }\end{array}$ & $4(12.5)$ \\
\hline $\begin{array}{l}\text { Incomplete due to home-based } \\
\text { continuation }\end{array}$ & $11(21.9)$ \\
\hline
\end{tabular}

\section{WHO-5 scores}

At SoS, WHO-5 well-being scores of all patients were highly distributed $(47.1 \pm 21.4)$ with scores ranging from 16 to 88 points. Differences between single questions were not reported (Figure 1A). At EoS, the well-being index improved to $69.7 \pm 15.1$ (range, 36-100). Most patients rated improved scores; only 4 patients had unchanged WHO-5 scores over time, and one patient reported a slight worsening of the well-being index. Changes between both evaluation times (difference $=$ EoS-SoS) reached from -4 to +80 index points. If single aspects of the WHO- 5 score were compared before and after yoga sessions, each item increased significantly $(\mathrm{p}<0.0005)$ (Figure 1B). Overall, WHO-5 score changes were highly significant $(\mathrm{p}<.001)$ for the entire patient cohort (Figure 2). This result means that the number of patients who reported having rare well-being ( $0-1$ point) reduced from about one third (33\%) of patients to approximately $5 \%$. In parallel, the percentage of patients reporting 4 and 5 points (frequent well-being) almost doubled during the time course of yoga sessions.

\section{Cofactors influencing treatment effects}

Treatment status, age, and diagnoses were compared to determine whether the observed effects could be attributed to one or more of various cofactors. A Kendall's Tau test was used because of the uneven distribution of patients within the groups. No significant differences on both SoS and EoS on WHO-5 scoring and over time between patients with continuous treatment and after therapy/during rehabilitation were found (data not shown).

In contrast, if age distribution was considered various findings were observed. Although the majority of the patients were between 40-60 years old, we were able to investigate the influence of age on the effects of yoga training. Interestingly, patients younger than 40 years (difference: $10.7 \pm 15.4$ ) and older than 60 years (difference: $16.0 \pm 12.1$ ) showed significantly less benefit $(\mathrm{p}<.005)$ from these mind-body approaches when compared with patients in the middle age group (difference: 28.0 20.6 ) (Figure 3). Similar observations were obtained for single items (not shown).

In addition, we analyzed if the WHO5 score at SoS relates to the potential for improvement at EoS or if the observed benefits were independent of initial well-being scores. As expected, patients with low initial WHO-5 scores had the highest potential for gaining higher wellbeing. We found a correlation between pre- and post-treatment scores. Patients with low initial scores benefited most from yoga training whereas patients with initial WHO-5 scores of $>75-80$ points did not show positive treatment effects as demonstrated by the cross-point between the regression line and equal benefit line (Figure 4).

Since our group performed a single arm trial without an untreated control group, we aimed to reduce the selection bias of patients. 
A

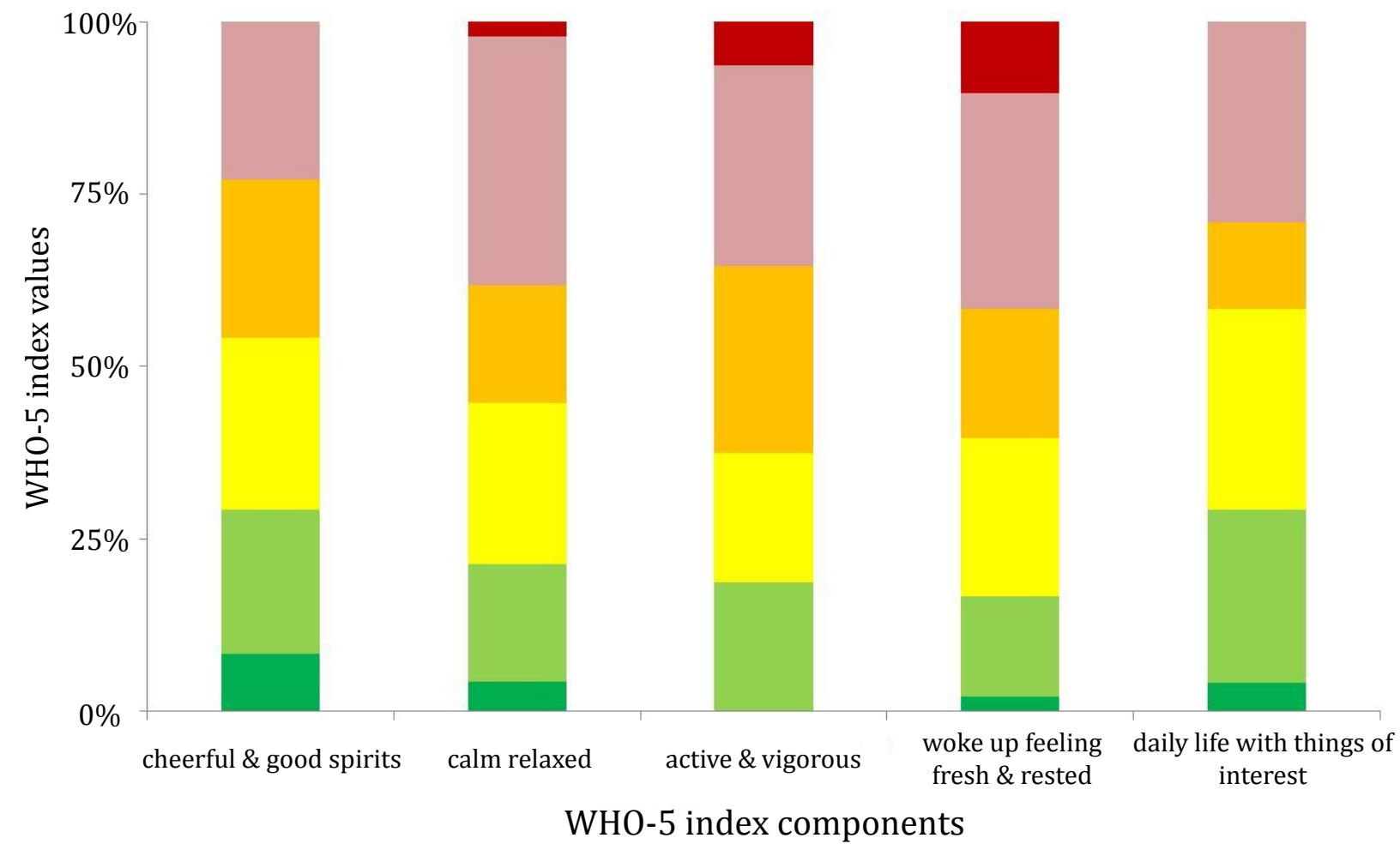

All of the time $\square$ Most of the time $\square$ More than half the time $\square$ Less than half the time $\square$ Some of the time $\square$ At no time

B

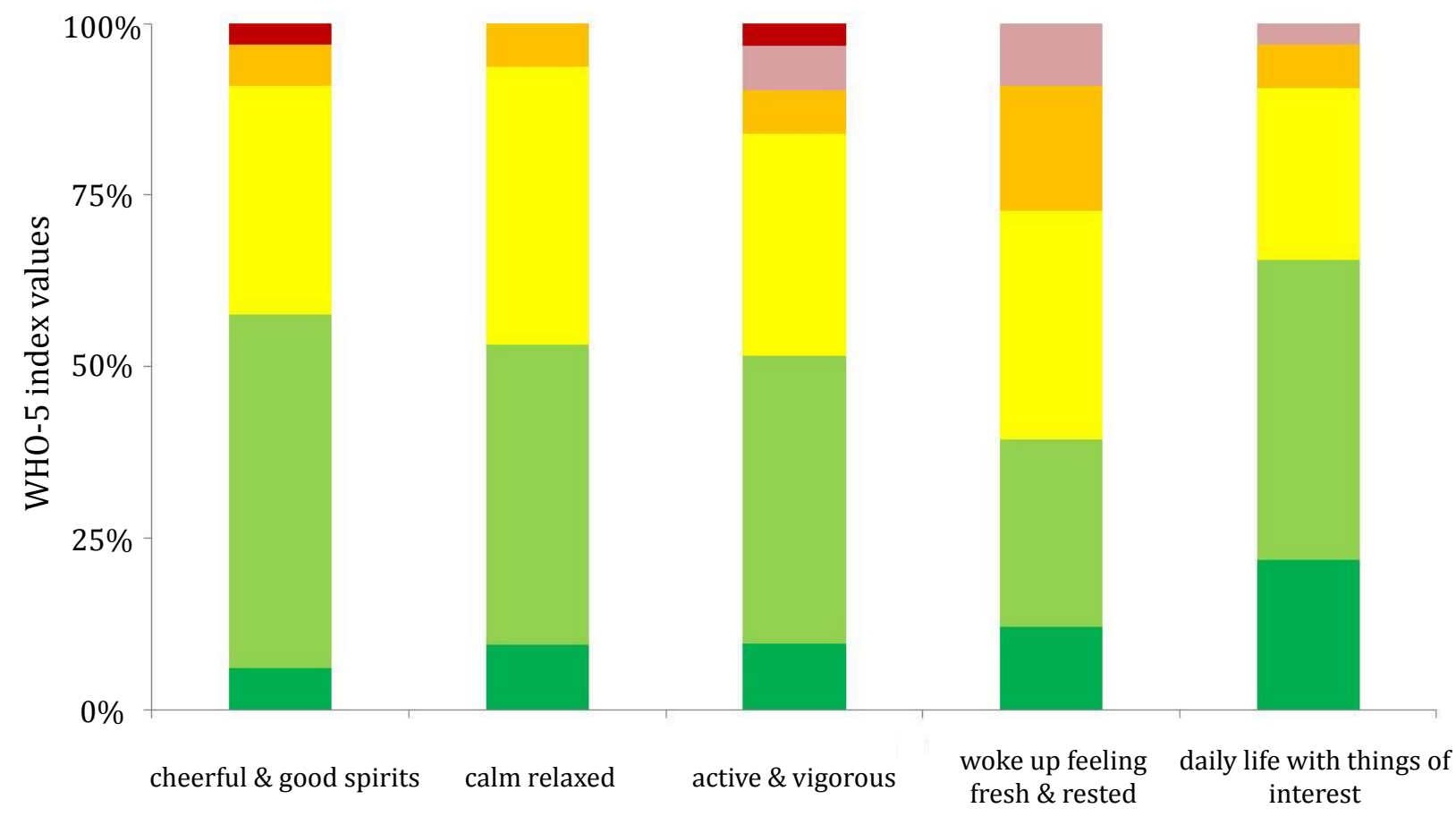

WHO-5 index components

All of the time $\square$ Most of the time More than half the time $\square$ Less than half the time $\square$ Some of the time $\square$ At no time

Figure 1. Distribution of self-reported well-being at (A) the start (SoS) and (B) the end of sessions (EoS). Percentage of patients who reported according to options for frequencies 


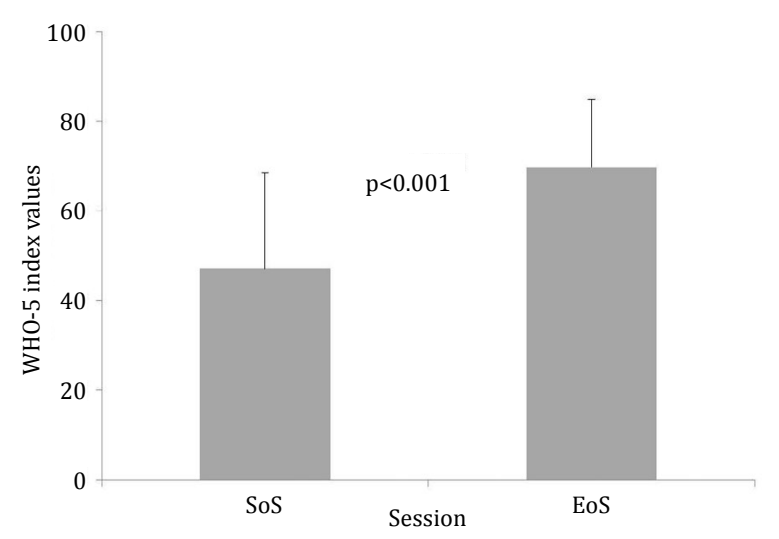

Figure 2. Differences in WHO-5 scores between the start (SoS) and end of sessions (EoS)

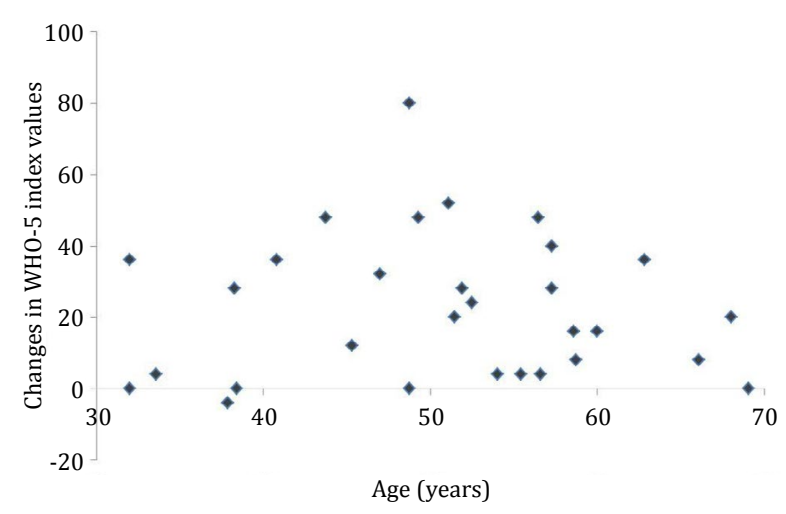

Figure 3. Age-related effects of yoga training on WHO-5 scores between the start ( $\mathrm{SoS}$ ) and end of sessions (EoS). Patients aged between 40-60 years old reported higher benefits

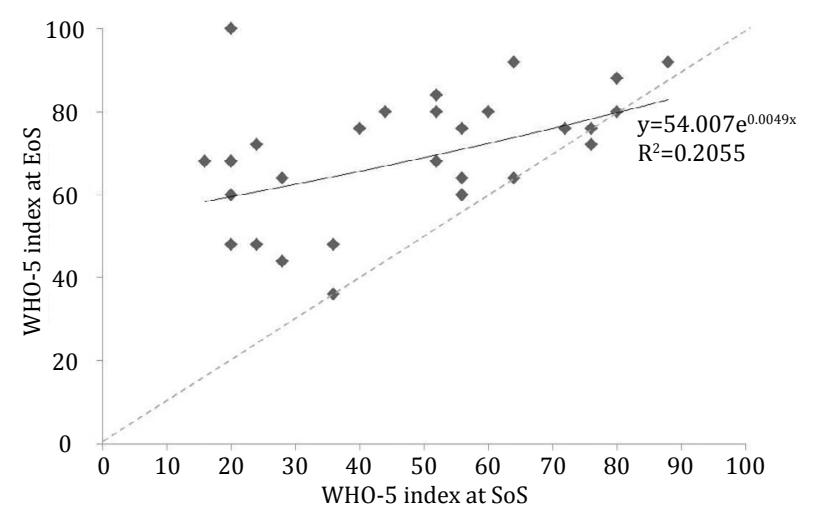

Figure 4. Pre- versus post-treatment WHO-5 scores. Regression line ( $\_$) crosses equal scoring line ( - - ) at WHO-5 value of approx. 80 points

In addition to the WHO-5 questionnaires, all participants were asked to provide feedback about information, scheduling, and organizational topics. The performance of supervised yoga lessons was rated exclusively with very positive scores (100\%). Information about training, its aims, and limitations for cancer patients were also considered as highly sufficient (93.3\% highly positive evaluation). Course management and scheduling were rated with slightly less satisfaction $(85.2 \%$ highly positive feedback). In light of these very high satisfaction rates, further analysis of these feedback factors in different patient groups was not done.

\section{DISCUSSION}

Our study showed that cancer patients seem to have a benefit from yoga exercises independent from their specific diagnosis. These benefits can be observed in all areas of well-being as defined by theWHO-5 evaluation tool. According to our results, positive effects are also not directly related to the scheduling of these complementary interventions during or after treatment. In our investigation, scheduling (group-based, once a week) only caused a few patients to drop out, but this was not related to yoga exposure. However, patients with the most relevant lack of well-being that can be corroborated by low WHO-5 index scores have the highest chance of achieving the most intensive improvement in their well-being indexes. It is noteworthy that very low WHO-5 scores were almost completely eliminated during the 10 sessions of specifically assisted yoga exercises and at EoS, about half of the patients reported (very) high scores.

In summary, our investigation of factors that influence the efficacy of yoga in different patient groups revealed that middle age (40-60 years) cancer patients appear to have higher benefits in addition to the above-mentioned patients with low WHO-5 indexes. This result is in line with our recently reported decisional preferences in cancer patients ${ }^{25}$ where age is a highly predictive factor that separates various patient groups according to their value framework in dealing with treatment opportunities. Interviews with patients during the training sessions confirmed that fear of inappropriate physical activity during cancer-specific treatment or in the early convalescence/rehabilitation phase is a highly burdensome problem, although this has not been systematically investigated in this study. 
Reduction of cancer treatment-related side effects, such as cognition deficits during chemotherapy, ${ }^{26}$ musculoskeletal problems under hormonal treatment, ${ }^{27}$ and menopausal symptoms ${ }^{28}$ by yoga approaches, were reported. In our trial, we focused on the five key aspects of the well-being of cancer patients as they were defined by the WHO. The short questionnaire had the advantage that it could be used repetitively during training sessions with sufficientacceptance by participants. A more comprehensive analysis would require questionnaires that are more sophisticated and/or other examinations, but according to our institutional experience with these types of programs, they are negatively associated with acceptance and response rates.

Studies on yoga benefits in cancer patients suffer from heterogeneity with various types of yoga interventions, duration, exposure, practices, and indications. ${ }^{29}$ For example, different types of yoga training, such as Bali Yoga, ${ }^{30,31}$ Vivekananda Yoga, ${ }^{32}$ and Hatha Yoga, ${ }^{33}$ among others might have a different influence on the observed effects, but evidence comparing various yoga types is still absent. In addition, the duration, schedules, and frequency of yoga support with or without specialized supervision differed in many published investigations to date. ${ }^{34}$ As in other studies, the positive effects in our investigation were found 3-6 months after starting yoga training. Long-term effects ${ }^{35}$ need to be more intensively investigated in future studies, and should also include both repetitive training periods and longterm supervision versus self-guided continuation of these exercises. Furthermore, various ways to offer yoga training were discussed, such as supervised group training as in our study, homebased efforts, ${ }^{36}$ or electronic support. ${ }^{37}$

The investigation of supportive treatment modalities at the end of or after cancer-specific treatment always interferes with regular convalescence, which is independent of interventions. Therefore, active comparator studies are recommended. However, the introduction of controlled designs in supportive treatment evaluation is not accepted in a sufficient manner by cancer patients even with very intensive recruitment efforts as our group has recently experienced. ${ }^{38}$ Decision preferences in these patients at a targeted time point have a strong influence ${ }^{25}$ that intensively interfere with trial participation. In our investigation, we used a single-institution approach without an untreated control group in a pre-post-design. The authors are aware of the limitations of this approach. Training groups were limited to small group training for adequate supervision by a specialized trainer who had experience with cancer patients. The authors acknowledge that these restrictions interfere with the generalization of the obtained results. Overall, general recommendations to include yoga into the treatment spectrum for breast cancer patients have been very recently provided by the American Cancer Society. ${ }^{21}$ Among others, yoga was recommended for anxiety/stress reduction, depression/mood disorders, and quality of life improvement. Details about the best yoga approach are not established yet. Our investigation provided additional evidence demonstrating that the yoga mind-body approach can be considered a very beneficial complementary treatment in cancer patients in general. This support should be offered to relevant patient groups independent of their cancer diagnosis and treatment status. Best effects can be expected in patients with low initial well-being and 40-60-year-old patients. In conclusion, yoga mind-body approaches can be considered as beneficial complementary treatment in relevant groups of cancer patients, especially if these patients suffer from low wellbeing during cancer treatment and are in the initial convalescence period.

\section{Conflict of Interest}

Jörg Haier is one of the editorial board members, but was not involved in the review or decision process of the article. A. Huda and C. Branss-Tallen get a grant from Krebsgesellschaft NRW.

\section{REFERENCES}

1. Ebede CC, Jang Y, Escalante CP. Cancer-related fatigue in cancer survivorship. Med Clin North Am. 2017;101(6):1085-97.

2. McNeely ML, Campbell KL, Rowe BH, Klassen TP, Mackey JR, Courneya KS. Effects of exercise on breast cancer patients and survivors: a systematic review and metaanalysis. CMAJ. 2006;175(1):34-41.

3. Rao RM, Vadiraja HS, Nagaratna R, Gopinath KS, Patil $\mathrm{S}$, Diwakar RB, et al. Effect of yoga on sleep quality and neuroendocrine immune response in metastatic breast cancer patients. Indian J Palliat Care. 2017;23(3):253-60. 
4. Kimura K. Yoga therapy in Japan. Int J Yoga Therap. 2017;27(1):127-9.

5. Milbury K, Kavanagh A, Meng Z, Chen Z, Chandwani KD, Garcia K, et al. Depressive symptoms and positive affect in Chinese and United States breast cancer survivors: a cross-cultural comparison. Support Care Cancer. 2017;25(7):2103-9.

6. Lipton L. Using yoga to treat disease: an evidence-based review. JAAPA. 2008;21(2):34-6.

7. Pascoe MC, Thompson DR, Ski CF. Yoga, mindfulnessbased stress reduction and stress-related physiological measures: a meta-analysis. Psychoneuroendocrinology. 2017;86:152-68.

8. Vadiraja HS, Rao RM, Nagarathna R, Nagendra HR, Patil $\mathrm{S}$, Diwakar RB, et al. Effects of yoga in managing fatigue in breast cancer patients: a randomized controlled trial. Indian J Palliat Care. 2017;23(3):247-52.

9. Rao RM, Raghuram N, Nagendra HR, Kodaganur GS, Bilimagga RS, Shashidhara HP, et al. Effects of a yoga program on mood states, quality of life, and toxicity in breast cancer patients receiving conventional Treatment: a randomized controlled trial. Indian J Palliat Care. 2017;23(3):237-46.

10. Chaoul A, Milbury K, Spelman A, Basen-Engquist K, Hall $\mathrm{MH}$, Wei $\mathrm{Q}$, et al. Randomized trial of Tibetan yoga in patients with breast cancer undergoing chemotherapy. Cancer. 2018;124(1):36-45.

11. Grégoire C, Bragard I, Jerusalem G, Etienne AM, Coucke P, Dupuis G, et al. Group interventions to reduce emotional distress and fatigue in breast cancer patients: a 9-month follow-up pragmatic trial. $\mathrm{Br} \mathrm{J}$ Cancer. 2017;117(10):1442-9.

12. Carson JW, Carson KM, Olsen MK, Sanders L, Porter LS. Mindful yoga for women with metastatic breast cancer: design of a randomized controlled trial. BMC Complement Altern Med. 2017;17:153.

13. Loudon A, Barnett T, Williams A. Yoga, breast cancerrelated lymphoedema and well-being: a descriptive report of women's participation in a clinical trial. J Clin Nurs. 2017;26(23-24):4685-95.

14. Ben-Josef AM, Chen J, Wileyto P, Doucette A, Bekelman J, Christodouleas J, et al. Effect of eischens yoga during radiation therapy on prostate cancer patient symptoms and quality of life: a randomized phase II trial. Int J Radiat Oncol Biol Phys. 2017;98(5):1036-44.

15. Milbury K, Mallaiah S, Mahajan A, Armstrong T, Weathers SP, Moss KE, et al. Yoga program for high-grade glioma patients undergoing radiotherapy and their family caregivers. Integr Cancer Ther. 2018;17(2):332-6.

16. Courneya KS, McKenzie DC, Reid RD, Mackey JR, Gelmon $\mathrm{K}$, Friedenreich CM, et al. Barriers to supervised exercise training in a randomized controlled trial of breast cancer patients receiving chemotherapy. Ann Behav Med. 2008; 35(1):116-22.

17. Rogers LQ Courneya KS, Verhulst S, Markwell SJ, McAuley E. Factors associated with exercise counseling and program preferences among breast cancer survivors. J Phys Act Health. 2008;5(5):688-705.

18. Courneya KS, Friedenreich CM, Quinney HA, Fields $\mathrm{AL}$, Jones LW, Vallance JK, et al. A longitudinal study of exercise barriers in colorectal cancer survivors participating in a randomized controlled trial. Ann Behav Med. 2005;29(2):147-53.
19. Buffart LM, van Uffelen JG, Riphagen II, Brug J, van Mechelen W, Brown WJ, et al. Physical and psychosocial benefits of yoga in cancer patients and survivors, a systematic review and meta-analysis of randomized controlled trials. BMC Cancer. 2012;12:559.

20. Cramer H, Lauche R, Klose P, Lange S, Langhorst J, Dobos GJ. Yoga for improving health-related quality of life, mental health and cancer-related symptoms in women diagnosed with breast cancer. Cochrane Database Syst Rev. 2017;1:CD010802.

21. Greenlee H, DuPont-Reyes MJ, Balneaves LG, Carlson LE, Cohen MR, Deng G, et al. Clinical practice guidelines on the evidence-based use of integrative therapies during and after breast cancer treatment. CA Cancer J Clin. 2017;67(3):194-232.

22. Carlson LE, Zelinski E, Toivonen K, Flynn M, Qureshi M, Piedalue KA, et al. Mind-body therapies in cancer: what is the latest evidence? Curr Oncol Rep. 2017;19(10):67.

23. Topp CW, Østergaard SD, Søndergaard S, Bech P. The WHO-5 Well-Being Index: a systematic review of the literature. Psychother Psychosom. 2015;84(3):167-76.

24. Staehr Johansen K: The use of well-being measures in primary health care - the DepCare project; in World Health Organization, Regional Office for Europe: WellBeing Measures in Primary Health Care - the DepCare Project. Geneva, World Health Organization, 1998, target 12, E60246.

25. Arnholdt J, Haier J. Cancer patients' preferences for therapy decisions can be grouped into categories and separated by demographic factors. J Cancer Res Clin Oncol. 2017;143(8):1573-84.

26. Galantino ML, Greene L, Daniels L, Dooley B, Muscatello L, O'Donnell L. Longitudinal impact of yoga on chemotherapy-related cognitive impairment and quality of life in women with early stage breast cancer: a case series. Explore (NY). 2012;8(2):127-35.

27. Peppone LJ, Janelsins MC, Kamen C, Mohile SG, Sprod LK, Gewandter JS, et al. The effect of YOCAS ${ }^{\circledR}$ yoga for musculoskeletal symptoms among breast cancer survivors on hormonal therapy. Breast Cancer Res Treat. 2015;150(3):597-604.

28. Cramer H, Rabsilber S, Lauche R, Kümmel S, Dobos G. Yoga and meditation for menopausal symptoms in breast cancer survivors-a randomized controlled trial. Cancer. 2015;121(13):2175-84.

29. Rao RM, Amritanshu R, Vinutha HT, Vaishnaruby S, Deepashree S, Megha M, et al. Role of yoga in cancer patients: expectations, benefits, and risks: a review. Indian J Palliat Care. 2017;23(3):225-30.

30. Lanctôt D, Dupuis G, Marcaurell R, Anestin AS, Bali M. The effects of the Bali Yoga Program (BYP-BC) on reducing psychological symptoms in breast cancer patients receiving chemotherapy: results of a randomized, partially blinded, controlled trial. J Complement Integr Med. 2016;13(4):405-12.

31. Lötzke D, Wiedemann F, Rodrigues Recchia D, Ostermann T, Sattler D, Ettl J, et al. Iyengar-Yoga compared to exercise as a therapeutic intervention during (Neo)adjuvant therapy in women with stage I-III breast cancer: health-related quality of life, mindfulness, spirituality, life satisfaction, and cancer -related fatigue. Evid Based Complement Alternat Med. 2016;5931816.

32. Milbury K, Mallaiah S, Lopez G, Liao Z, Yang C, Carmack 
C, et al. Vivekananda Yoga program for patients with advanced lung cancer and their family caregivers. Integr Cancer Ther. 2015;14(5):446-51.

33. Chen N, Xia X, Qin L, Luo L, Han S, Wang G, et al. Effects of 8-week Hatha Yoga training on metabolic and inflammatory markers in healthy, female Chinese subjects: a randomized clinical trial. BioMed Res Int. 2016;2016:5387258

34. Hughes DC, Darby N, Gonzalez K, Boggess T, Morris RM, Ramirez AG. Effect of a six-month yoga exercise intervention on fitness outcomes for breast cancer survivors. Physiother Theory Pract. 2015;31(7):451-60.

35. Amritanshu RR, Rao RM, Nagaratna R, Veldore VH, Rani $\mathrm{MR}$, Gopinath KS, et al. Effect of long-term yoga practice on psychological outcomes in breast cancer survivors.
Indian J Palliat Care. 2017;23(3):231-6.

36. Komatsu H, Yagasaki K, Yamauchi H, Yamauchi $T$, Takebayashi T. A self-directed home yoga programme for women with breast cancer during chemotherapy: A feasibility study. Int J Nurs Pract. 2016;22(3):258-66.

37. Lengacher CA, Reich RR, Ramesar S, Alinat CB, Moscoso M, Cousin L, et al. Feasibility of the mobile mindfulness-based stress reduction for breast cancer (mMBSR(BC)) program for symptom improvement among breast cancer survivors. Psychooncology. 2017;27(2):524-31

38. Schweer C, Doering S, Haier J, Heuft G, Fritz F, Dugas M, et al. Psychooncological interventions - what do cancer patients aged 60 years or older wish for? Z Psychosom Med Psychother. 2011;57(3):223-32. 